\title{
Difusão do processo educativo para a qualificação da gestão do SUS em saúde bucal:
}

\section{Uma análise espacial}

\author{
Diffusion of the educational process for the qualification of SUS management in oral health: A \\ spatial analysis
}

Difusión del proceso educativo para la calificación de la gestión del SUS en salud bucal: Un análisis espacial

\section{Resumo}

O artigo avalia a difusão do processo educativo para a qualificação da gestão do SUS em Saúde Bucal no Paraná, realizado em quatro ciclos de capacitação, de 2012 a 2019. Utilizou-se de um estudo exploratório espacial, do tipo ecológico, tendo como base teórica a teoria da difusão de inovação. Foram elaborados mapas utilizando bancos de dados sobre as edições do curso oferecido para os Municípios e Regionais de Saúde que aderiram e concluíram os quatro ciclos. No total do processo, realizaram a inscrição voluntária 1.304 profissionais de saúde (equipe multiprofissional) de 321 municípios (80\% dos municípios do Paraná), e 877 participantes de 259 municípios concluíram o Curso. Esta iniciativa apresentou vantagem relativa, compatibilidade, observabilidade, experimentabilidade e baixa complexidade, além de utilizar canais de comunicação adequados e ter o favorecimento do sistema social e o esforço dos agentes de mudança no processo de trabalho. Conclui-se que o Curso de Qualificação da Gestão do SUS em Saúde Bucal apresentou as características da Teoria da Difusão de Inovação, configurando-se um processo inovador em educação profissional com a adesão da maior parte dos municípios do Estado do Paraná, reforçando o papel da importância da integração ensino/serviço em benefício da população.

Palavras-chave: Sistemas de informação geográfica; Avaliação educacional; Difusão de inovações.

\begin{abstract}
The article evaluates the diffusion of the educational process for the qualification of SUS management in Oral Health in Paraná, conducted in four training cycles, from 2012 to 2019. It was used an exploratory study, of the ecological type. Maps were prepared using Course databases and QGIS Software for the Municipalities and Regional Health that adhered to and completed the four cycles, analyzing the diffusion of innovation through Rogers' Diffusion of Innovation Theory. In total, 1,304 health professionals (multidisciplinary team) from 321 municipalities (80\% of the municipalities of Paraná) volunteered to enroll, and 877 participants from 259 municipalities completed the course. This initiative presented relative advantage, compatibility, observability, experimentability and low complexity, besides using adequate communication channels and having the favor of the social system and the effort of agents of change in the process. It is concluded that the SUS Management Qualification Course in Oral Health presented the characteristics of the Innovation Diffusion Theory, setting up an innovative process in professional education with spread in the State of Paraná, reinforcing the role of the importance of teaching / service for the benefit of the population.
\end{abstract}

Keywords: Geographic information systems; Educational assessment; Diffusion of innovations. 


\begin{abstract}
Resumen
El artículo evalúa la difusión del proceso educativo para la calificación de la gestión del SUS en Salud Bucal en Paraná, realizado en cuatro ciclos formativos, de 2012 a 2019. Se utilizó como base teórica un estudio exploratorio espacial, de tipo ecológico. la teoría de la difusión de la innovación. Se elaboraron mapas utilizando bases de datos de las ediciones del curso ofrecidas a los Municipios y Regiones de Salud que se incorporaron y completaron los cuatro ciclos. En el proceso total, 1.304 profesionales de la salud (equipo multiprofesional) de 321 municipios ( $80 \%$ de los municipios de Paraná) completaron el Curso y 877 participantes de 259 municipios completaron el curso. Esta iniciativa tuvo relativa ventaja, compatibilidad, observabilidad, experimentación y baja complejidad, además de utilizar canales de comunicación adecuados y favorecer el sistema social y los esfuerzos de los agentes de cambio en el proceso de trabajo. Se concluye que el Curso de Calificación Gerencial del SUS en Salud Bucal presentó las características de la Teoría de la Difusión de la Innovación, configurando un proceso innovador en la formación profesional con la adhesión de la mayoría de los municipios del Estado de Paraná, reforzando el rol la importancia de la integración docencia / servicio en beneficio de la población.
\end{abstract}

Palabras clave: Sistemas de información geográfica; Evaluación educativa; Difusión de las innovaciones.

\title{
1. Introdução
}

A Política Nacional de Saúde Bucal aponta a necessidade de reorganização do processo de desenvolver ações e serviços de saúde, bem como aprimorar a gestão do processo de trabalho por parte dos profissionais, garantindo a qualificação da atenção à saúde com resolutividade e integralidade (Brasil, 2004). No entanto, a gestão dos serviços de saúde bucal no âmbito do SUS permanece permeada por inúmeros conflitos e contradições, destacando neste âmbito os aspectos relativos aos recursos humanos, com baixa qualificação e formação dos profissionais para trabalhar na promoção e prevenção em saúde bucal, planejamento e diagnóstico do território (Frazão \& Narvai, 2009).

Devido ao processo de descentralização das ações de saúde no Brasil, com ênfase na municipalização, os profissionais passam a ter um papel importante no protagonismo da gestão local e a necessidade de qualificar-se para utilização de ferramentas como o diagnóstico, planejamento e a avaliação, além do uso de indicadores para gerenciar o processo de trabalho e do cuidado (Brasil, 2006). A gestão deve fomentar o trabalho interprofissional e intersetorial, atuando sobre os determinantes sociais do processo saúde-doença e incorporação de práticas baseadas em evidências de efetividade (Kusma, Moysés \& Moysés, 2012).

No entanto, deve-se destacar que os municípios e suas unidades de saúde não podem ser tomados como entidades isoladas, mas parte de um sistema com inúmeras conexões com outros entes federados e entre estabelecimentos de maior ou menor complexidade. Neste sentido a descentralização das ações de saúde pressupõe o estabelecimento de vínculos entre a rede de saúde e a rede de cidades, que possuem complementariedades e hierarquias (Duarte et al., 2015). A Teoria da Difusão de Inovação permite que se identifique alguns padrões na dinâmica espacial de espalhamento e implementação de uma determinada prática ou tecnologia, que possui trajetórias, centralidades, barreiras e limites de adoção.

No processo de qualificação dos profissionais de saúde destaca-se o papel das Instituições de Ensino Superior (IES) tanto na formação durante a graduação quanto para fomentar a educação permanente dos profissionais em seus postos de trabalho com uma possível reconfiguração da prática (Rangel et al., 2012). A Universidade Estadual de Maringá (UEM) propôs, através do Curso de Extensão "Qualificação da gestão do SUS em Saúde Bucal", articular as relações entre a comunidade acadêmica e a sociedade, na perspectiva de transformação social e qualificar os profissionais do SUS. Segundo as propostas da Política Nacional de Extensão Universitária (PROEX, 2012), o processo de avaliação deve mensurar a contribuição oferecida pelas ações extensionistas para a produção e transmissão do conhecimento e transformação social, bem como a amplitude de difusão das ações. Não obstante, a literatura mostra que ainda é recente o processo de avaliação das ações universitárias com relação à eficiência e eficácia (Arroyo \& Rocha, 2010). 
As Universidades devem exercer seu protagonismo na geração do conhecimento, aprendizagem e inovação, com utilização de novas tecnologias de informação, substituição de aulas expositivas por metodologias ativas de ensinoaprendizagem, como estímulo aos trabalhos em equipe e o uso do ensino à distância (Masseto, 2004), bem como o rompimento da lógica atual da maneira que os indivíduos aprendem, numa inovação de paradigma (Christensen et al., 2012). A inovação deve ser um processo habilitado por uma gestão bem estruturada, coordenação atuante (Bessant et al., 2005) e tornam-se efetivas quando há o processo de difusão e utilização adequada de seus elementos condicionantes, auxiliando a organização atingir seus objetivos (Giacomini Filho, Goulart \& Caprino, 2007).

Nas diversas áreas de conhecimento, existem vários conceitos para a inovação (Brasil, 2018). Para o presente estudo, considerou-se a inovação e a difusão como conceitos diferentes e interligados, de acordo com a teoria de Difusão de Inovação de Rogers (2003), segundo ela, a difusão é "o processo pelo qual uma inovação é difundida por meio de canais ao longo do tempo, dentre membros de um sistema social. É um tipo especial de comunicação, na qual as mensagens são ligadas a novas ideias". Além disso, é um processo pelo qual mudanças ocorrem na estrutura e função de um sistema social, trazendo impactos sociais.

Desta forma, o objetivo deste estudo foi avaliar a qualificação da gestão do SUS em Saúde Bucal no Paraná como um processo de difusão de inovação, o que depende tanto da incorporação de novas tecnologias, quanto da estrutura existente do setor saúde, o que vai influenciar a velocidade do processo de difusão e a adesão dos participantes a essas inovações. Nesse sentido, esse artigo explorou as categorias de análise adaptadas de Rogers (2003) de vantagem relativa, compatibilidade, complexidade, experimentabilidade e observabilidade para avaliar o processo de difusão de uma iniciativa de educação.

\section{Metodologia}

Foi realizado um estudo exploratório e ecológico espacial com dados secundários (IBGE, SUS e IPARDES) e primários (CCQG). O estudo ecológico espacial consiste em analisar diferentes localidades na mesma época. O estudo com delineamento ecológico tem a capacidade de gerar hipótese espacial (Morgenstern, 1995). Foi usada análise espacial como técnica de tratamento dos dados. As Regionais de Saúde e os municípios do Estado do Paraná são as unidades geográficas de análise. O estudo teve cunho quantitativo e qualitativo, utilizando a análise espacial como técnica de tratamento dos dados coletados ao longo dos ciclos do curso de extensão "Qualificação da gestão do SUS em Saúde Bucal". Esses dados foram georreferenciados segundo municípios e analisados por Regionais de Saúde. Em relação ao processo de difusão da inovação, existem cinco elementos condicionantes no processo de adesão da inovação que devem ser mencionados: I) as características percebidas da inovação (vantagem relativa, compatibilidade, complexidade, experimentabilidade e observabilidade); II) o tipo de decisão (opcional, coletiva e autoritária); III) os canais de comunicação; IV) as características da unidade decisória; V) a extensão dos esforços dos agentes de mudança (Rogers, 2003).

Indicadores quantitativos e qualitativos sobre esses elementos foram gerados a partir de dados coletados durante os ciclos de execução do curso e por meio de dados socioeconômicos organizados segundo municípios.

\subsection{Local do estudo}

O Estado do Paraná é uma das 27 unidades federativas do Brasil e está localizado na Região Sul. A estimativa populacional para o ano de 2019 é de 11.433.957 (IBGE, 2012). Neste estudo utilizou-se o estado do Paraná dividido em 399 municípios e 22 Regionais de Saúde (SESA, 2018). Segundo o censo demográfico do Instituto Brasileiro de Geografia e Estatística (IBGE, 2012) o índice de desenvolvimento humano (IDH) do estado era 0,749, sendo o quinto maior do país. 


\subsection{Curso de Qualificação da Gestão do SUS em Saúde Bucal}

O Curso foi elaborado pelo Departamento de Odontologia da UEM e aprovado pelo Ministério da Saúde no ano de 2010, através do convênio 1.333/2010. A primeira etapa do projeto consistiu em realizar um levantamento da literatura sobre as condutas para a qualificação da gestão em saúde bucal visando a elaboração de um instrumento diagnóstico da gestão local, representando a base de estudo e dos conteúdos do Curso de "Qualificação da Gestão do SUS em Saúde Bucal". Nesta fase, buscou-se um alinhamento conceitual entre as três esferas do governo, convergindo para um norte comum da gestão da saúde bucal. Vale ressaltar que este instrumento é uma ferramenta para o diagnóstico situacional, cuja estrutura conceitual pode ser aplicada em qualquer município, o que tem possibilitado a reflexão e a elaboração de propostas de intervenção para a melhoria da gestão e da atenção à saúde bucal; entretanto, não tem finalidade avaliativa e de comparação entre municípios e equipes de saúde bucal (Fujimaki et al., 2019).

A proposta pedagógica do Curso de "Qualificação da Gestão do SUS em Saúde Bucal" foi estruturada no formato semipresencial. O material didático está organizado em seis módulos e foi construído e acomodado na plataforma do Google Drive, a qual permite a criação de documentos compartilhados, organização de formulários, sendo um repositório de arquivos digitais. O conteúdo teórico dos módulos foi elaborado com a inclusão de "hiperlinks" para artigos, vídeos e outros materiais instrucionais, o que permitiu trabalhar utilizando Tecnologias de Informação e Comunicação (TIC) para o "e-learning" e "blended-learning", tendo sido compartilhado com os alunos no Google Drive ou encaminhado por e-mail. Assim, considerando a necessidade do alinhamento conceitual e o do envolvimento de gestores e equipes de saúde para um norte comum na melhoria da saúde bucal, o Curso foi pensado, tendo a seguinte estruturação, baseado em ciclos formativos:

$1^{\circ}$ ciclo: Capacitação da equipe de saúde bucal da Secretaria Estadual de Saúde do Paraná (SESA) e 22 coordenadores regionais de saúde bucal do Paraná (etapa realizada em 2012);

$2^{\circ}$ ciclo: Capacitação de coordenadores municipais de saúde bucal (etapa realizada em 2013-2014);

$3^{\circ}$ ciclo: Capacitação das equipes de saúde bucal, incluindo Cirurgiões Dentistas (CD), Técnicos em Saúde Bucal (TSB), Auxiliares de Saúde Bucal (ASB) do Estado do Paraná (etapa realizada em 2015-2016).

$4^{\circ}$ ciclo: Capacitação das equipes de saúde (incluindo os membros das equipes multiprofissionais) (etapa realizada em 2017-2019).

Durante o curso, os participantes realizaram o diagnóstico da gestão local e planejamento, apresentaram e implementaram propostas de intervenções, que foram descritas nos respectivos Trabalhos de Conclusão do Curso (TCC), sendo a entrega deste um requisito para conclusão (Fujimaki et al., 2019).

\subsection{Aquisição de dados e variáveis}

Neste estudo foi utilizado a base de dados da Coordenação do Curso de Qualificação da Gestão do SUS em Saúde Bucal. No início de cada ciclo, todos os participantes (alunos e multiplicadores) preencheram um formulário on-line no Google Form com perguntas relacionadas à categoria profissional, município pertencente e outras informações pertinentes à condução do Curso. Este formulário foi encaminhado por e-mail pela Coordenação do Curso. Ao final de cada ciclo, todos preencheram um formulário de avaliação final do curso, com perguntas abertas e fechadas sobre sua percepção em relação às mudanças realizadas com o curso. Além disso, foram consultados os documentos do TCC para comprovação da conclusão. Todos os dados foram coletados por um único pesquisador em maio de 2019.

Para permitir a análise do contexto da participação dos municípios e seus possíveis condicionantes da difusão, foi utilizado o Índice de Desenvolvimento Humano Municipal (IDH-M) dos municípios do Estado do Paraná que foi obtido do Instituto Paranaense de Desenvolvimento Econômico e Social - IPARDES (2010). 
Para a análise dos elementos condicionantes da difusão de inovação, foi realizada uma pesquisa documental em capítulos de livros publicados sobre o Curso de Qualificação da Gestão do SUS em Saúde Bucal (Fujimaki et al., 2019), conteúdo teórico dos 6 módulos do Curso e relatórios de projetos de pesquisa realizados (Tzusuki (2017); Galbiati (2018)).

\subsection{Análise de dados}

Foram analisadas as seguintes variáveis do Curso: Regionais de Saúde, Municípios, Participantes e Multiplicadores. Para cada ciclo, foi calculada a taxa de adesão voluntária ao Curso, o número de participantes e multiplicadores, bem como a taxa de conclusão, ou seja, os participantes que realizaram a entrega do TCC. Os dados quantitativos foram tabulados segundo o município de realização do curso e georreferenciado para a produção de mapas temáticos de indicadores. Para a construção dos mapas de adesão e conclusão foi utilizado o Sistema de Informação Geográfico (SIG), QGIS versão 3.4.6.

Os dados qualitativos, coletados por meio de campos do formulário de avaliação foram organizados segundo as categorias de análise propostas por Rogers (2003) de vantagem relativa, compatibilidade, complexidade, experimentabilidade e observabilidade. Além dessas foram analisados aspectos de tipo de decisão, canais de comunicação utilizados, a natureza do sistema social, e a extensão dos esforços dos agentes de mudança.

\section{Resultados}

Verificou-se que no primeiro ciclo (2012), todas as 22 Regionais de Saúde (100\%) participaram com a adesão dos seus coordenadores regionais de Saúde Bucal (Figura 1).

Figura 1. Mapa do Estado do Paraná dividido em Regionais de Saúde. Maringá-PR, 2019.

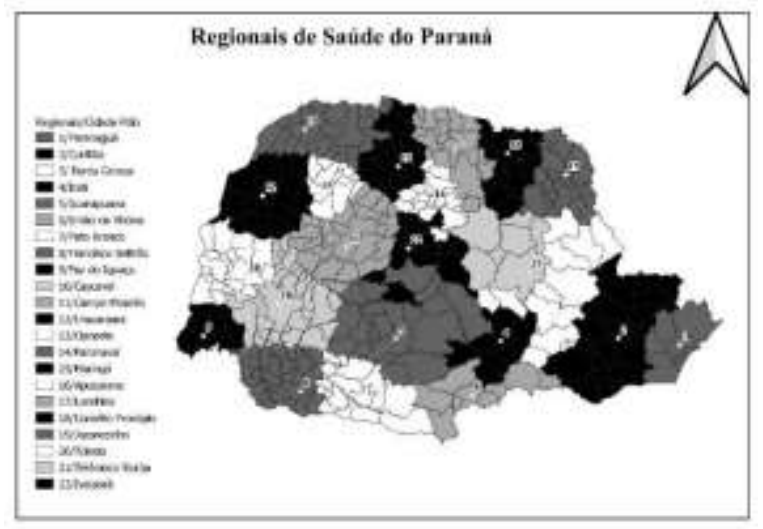

Fonte: Autores.

No ciclo seguinte (2013-2014), 250 municípios (63\%) realizaram adesão ao Curso, sendo que as Regionais de Curitiba, Ponta Grossa, Guarapuava, União da Vitória, Pato Branco, Foz do Iguaçu, Umuarama, Paranavaí, Maringá, Apucarana, Cornélio Procópio, Jacarezinho, Telêmaco Borba e Ivaiporã tiveram mais de 70\% de adesão de seus municípios, destacando 100\% de adesão na Regional de Foz do Iguaçu, Jacarezinho e Telêmaco Borba. Já as Regionais de Paranaguá, Irati, Francisco Beltrão, Cascavel, Cianorte e Toledo tiveram menos de 10\% de adesão de seus municípios.

No terceiro ciclo (2015-2016), 84 municípios (21\%) aderiram ao Curso, sendo que as Regionais de Paranaguá, Guarapuava, Cascavel, Campo Mourão, Umuarama, Cianorte, Paranavaí, Londrina, Cornélio Procópio, Toledo, Telêmaco Borba e Ivaiporã tiveram menos de 10\% de adesão de seus municípios. As Regionais de Francisco Beltrão e Irati tiveram mais de $70 \%$ de adesão de seus municípios. 
No quarto ciclo (2017-2018) 161 (40\%) municípios aderiram ao Curso. Sendo que as Regionais de Paranaguá, Guarapuava e Francisco Beltrão tiveram a adesão de mais de 70\% dos municípios, destacando 100\% na Regional de Francisco Beltrão. Já as Regionais de Ponta Grossa, Cascavel, Paranavaí e Jacarezinho tiveram menos de 10\% de participação de seus municípios.

As Regionais de Curitiba, Irati, Guarapuava, União da Vitória, Pato Branco, Francisco Beltrão, Foz do Iguaçu, Umuarama, Maringá, Apucarana, Jacarezinho, Telêmaco Borba e Ivaiporã tiveram mais de $90 \%$ de adesão de seus municípios em pelo menos um ciclo. Em contrapartida, as Regionais de Cascavel e Toledo tiveram menos de 30\% dos municípios que aderiram a pelo menos um ciclo.

Dos 399 municípios do Paraná, 321 municípios (80\%) realizaram adesão ao Curso em pelo menos um ciclo de formação, sendo que 160 municípios (40\%) realizaram apenas um ciclo, 148 municípios (37\%) realizaram dois ciclos e 13 municípios (4\%) realizaram adesão nos três ciclos (Figura 2a).

Em relação à conclusão do Curso, no primeiro ciclo participaram e concluíram todos os 22 coordenadores regionais de Saúde Bucal (cidades polo) realizando o TCC. No segundo ciclo, foram 197 municípios (49\% dos municípios do Estado e $78 \%$ dos que aderiram ao Curso) e no terceiro ciclo foram 55 municípios (14\% dos municípios do Estado e 65\% dos que aderiram ao Curso). Já no quarto ciclo, 103 realizaram o TCC (26\% dos municípios do Estado e $64 \%$ dos que aderiram ao Curso). No Paraná, nos três ciclos 259 municípios realizaram o TCC (65\% dos municípios do Estado). Ou seja, 80\% dos municípios que aderiram e 877 profissionais, realizaram o TCC contendo um diagnóstico situacional, planejamento, além do relato da implementação de intervenções em sua realidade local (Figura 2b).

Figura 2A. Mapa da adesão nos três ciclos ao Curso de Qualificação da Gestão do SUS em Saúde Bucal. Maringá-PR, 2019; Figura 2B. Mapa cumulativo dos municípios que concluíram o Curso de Qualificação da Gestão do SUS em Saúde Bucal por ciclos. Maringá-PR, 2019.
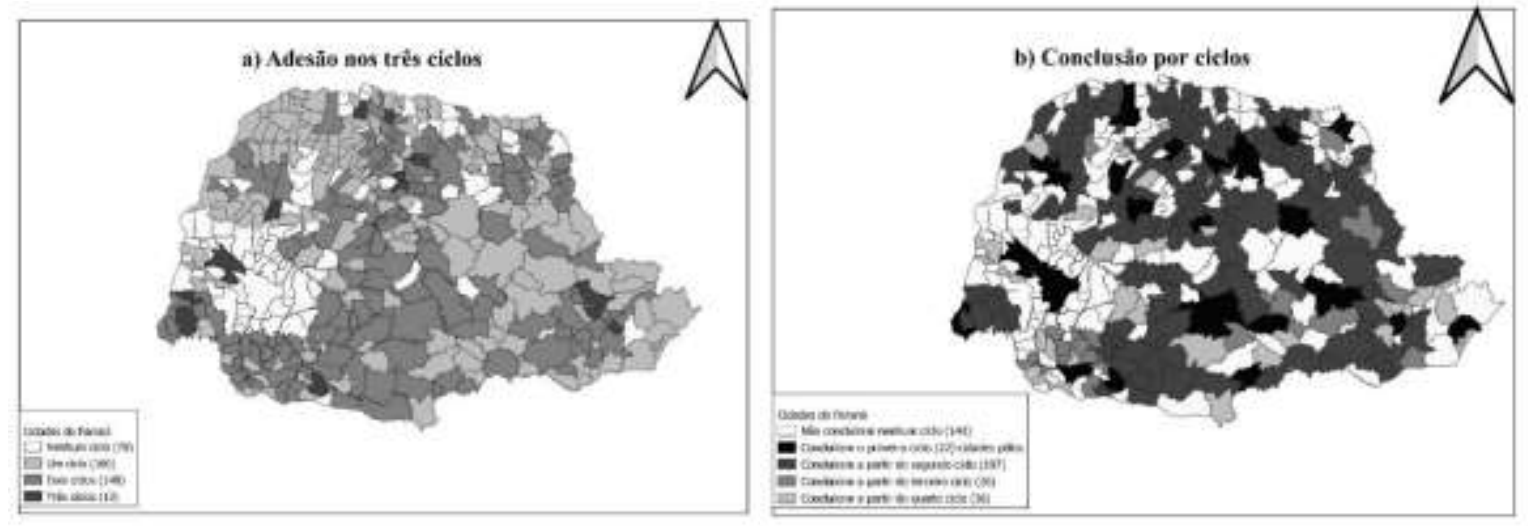

Fonte: próprio Fonte: Autores.

O Curso atingiu $100 \%$ dos municípios com IDH-M baixo $(0,5$ a 0,599$)$ e $69 \%$ dos com IDH-M médio $(0,6$ a 0,699$)$ (Figura 3), sendo menor a adesão de municípios com IDH alto, que em geral coincidem com cidades de maior porte, mais populosas e com ofertas de serviços. 
Research, Society and Development, v. 10, n. 8, e34410817460, 2021

(CC BY 4.0) | ISSN 2525-3409 | DOI: http://dx.doi.org/10.33448/rsd-v10i8.17460

Figura 3. Mapa dos municípios que realizaram o curso e a vulnerabilidade social. Maringá-PR, 2019.

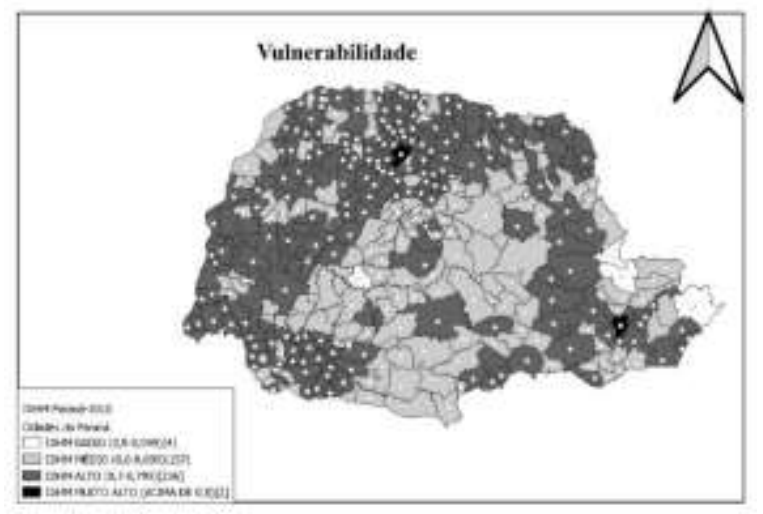

Fonte: Autores.

Na Tabela 1 estão apresentados os elementos condicionantes da difusão, adaptados de Moore e Benbasat (1991). 
Tabela 1. Elementos condicionantes da difusão de inovação.

Elementos

1.) Características da inovação

1.1) Vantagem Relativa

\begin{tabular}{|c|c|}
\hline 1.2)Compatibilidade & $\begin{array}{l}\text { O Curso estava alinhado à missão e objetivos do SUS, baseado nos princípios e diretrizes e norteada pelas Normativas vigentes e atualizada } \\
\text { do SUS. }\end{array}$ \\
\hline 1.3)Complexidade & $\begin{array}{l}\text { O Curso é fácil de implementar, pois ocorre em módulos. A implementação ocorre gradualmente e de acordo com a realidade local e } \\
\text { individual. Os custos são baixos, pois o material didático é disponibilizado de forma digital. }\end{array}$ \\
\hline 1.4)Experimentabilidade & $\begin{array}{l}\text { Os participantes primeiramente fazem o Curso e posteriormente são convidados a serem multiplicadores. Existiram } 95 \text { multiplicadores } \\
\text { voluntários nos } 4 \text { ciclos. }\end{array}$ \\
\hline 1.5) Observabilidade & $\begin{array}{l}\text { Consegue-se observar se os profissionais estão satisfeitos com o Curso, pela motivação dos participantes e multiplicadores, além da taxa } \\
\text { conclusão de } 80 \% \text {. Numa pesquisa realizada com participantes do } 3^{\circ} \text {. Ciclo, } 89,3 \% \text { afirmaram que o curso estimulou-os a buscar mais } \\
\text { informações para melhorar sua atuação como servidor público; } 95,6 \% \text { apontaram mudanças no interesse em buscar evidências para a prática } \\
\text { e/ou nas formas de atuar com prevenção e promoção de saúde e/ou no entendimento do papel do servidor no SUS (Galbiati, 2018). Relato de } \\
\text { um multiplicador: "[houveram] grandes mudanças na prática... é muito gratificante perceber que muda a visão do serviço, do entendimento } \\
\text { das realidades, das dificuldades e todo o potencial motivacional em criar novos modelos, novas formas de ver, sentir e encarar os desafios da } \\
\text { saúde bucal" (Tzusuki, 2017). }\end{array}$ \\
\hline 2) Tipo de decisão & A adesão foi voluntária, com $100 \%$ dos participantes e multiplicadores, nos 4 ciclos. \\
\hline $\begin{array}{l}\text { 3) Canais de } \\
\text { Comunicação }\end{array}$ & $\begin{array}{l}\text { Foram utilizados meios de comunicação adequados, uma vez que foi utilizada a comunicação interpessoal com gestores e comunicação } \\
\text { interinstitucional por meio de e-mails da UEM e da SESA. Foram realizadas reuniões presenciais no início e no final de cada ciclo com os } \\
\text { gestores para o planejamento e avaliação. E também foram realizadas reuniões por videoconferência com os multiplicadores antes de cada } \\
\text { módulo no quarto ciclo. }\end{array}$ \\
\hline
\end{tabular}

O Curso ofereceu benefício para o serviço e/ou profissional, pois oportunizou a vivência da educação permanente, fomentou pen samento crítico-reflexivo e transformou as práticas de trabalho em 321 municípios e 1304 profissionais que conheceram o Instrumento Diagnóstico. E pelo depoimento de um multiplicador: "Já percebi profissionais transformando sua posição frente à abordagem dos textos. Outros profissionais valorizando o conteúdo e trazendo junto aos obstáculos do dia a dia, as soluções. Por exemplo, uma equipe já planejando e programando horário diferenciado de atendimento para melhorar acesso de usuários trabalhadores" (Tzusuki, 2017).

Curso estava alinhado à missão e objetivos do SUS, baseado nos princípios e diretrizes e norteada pelas Normativas vigentes e atualizada

O Curso é fácil de implementar, pois ocorre em módulos. A implementação ocorre gradualmente e de acordo com a realidade local e Os participantes primeiramente fazem o Curso e posteriormente são convidados a serem multiplicadores. Existiram 95 multiplicadores 
Research, Society and Development, v. 10, n. 8, e34410817460, 2021

(CC BY 4.0) | ISSN 2525-3409 | DOI: http://dx.doi.org/10.33448/rsd-v10i8.17460

\begin{tabular}{l|l}
\hline $\begin{array}{l}\text { 4) Natureza do sistema } \\
\text { social }\end{array}$ & $\begin{array}{l}\text { O sistema social favoreceu a difusão, com parcerias com as três esferas de governo: Federal (Ministério da Saúde), Estadual (SESA) e } \\
\text { Municipal (Coordenadores Municipais). }\end{array}$ \\
\hline 5) Extensão dos esforços & $\begin{array}{l}\text { Os agentes de mudança ajudaram no processo de difusão, pois os Coordenadores Regionais (100\%) aderiram ao primeiro ciclo e foram } \\
\text { multiplicadores nos seguintes, formando novos multiplicadores. }\end{array}$ \\
\hline
\end{tabular}

Fonte: Elaboração própria. Pesquisa documental em capítulos de livros publicados, conteúdo teórico dos 6 módulos do Curso e relatórios de projetos de pesquisa realizados. 
Em relação ao quantitativo e perfil dos participantes, o primeiro ciclo iniciou com três multiplicadores que capacitaram 22 coordenadores regionais, o segundo ciclo foram 20 multiplicadores que capacitaram 384 profissionais (composto por coordenadores municipais de saúde bucal e profissionais da equipe de saúde bucal), e no terceiro ciclo 17 multiplicadores capacitaram 262 profissionais (composto por coordenadores de saúde bucal, CD, ASB e TSB). Em relação ao quarto ciclo, 55 multiplicadores capacitaram 636 profissionais (incluindo equipe multiprofissional, enfermeiro, Agentes Comunitários de Saúde (ACS), Agentes de Combate às Endemias (ACE), entre outros profissionais da Atenção Primária) (figura 4).

Figura 4. Adesão de alunos e multiplicadores por ciclos de capacitação. Maringá-PR, 2019.

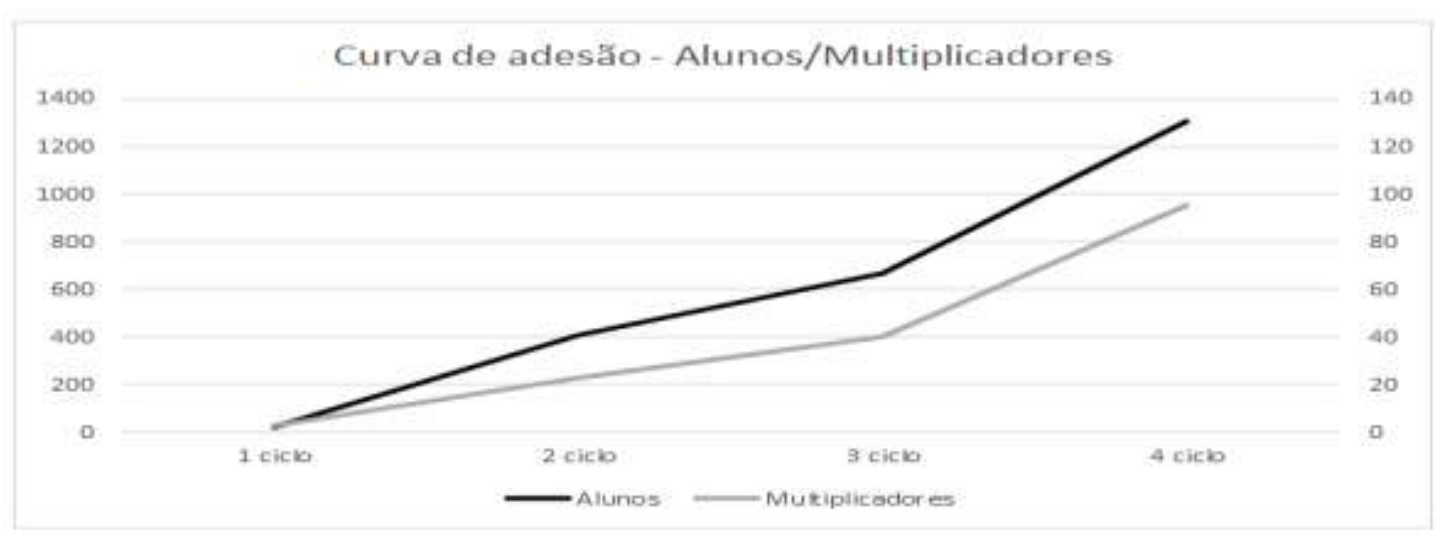

Fonte: Elaboração própria. Pesquisa na base de dados da Coordenação do Curso de Qualificação da Gestão do SUS em Saúde Bucal.

Percebe-se pela figura um processo acumulativo de adesão dos municípios ao curso ao longo dos seus ciclos.

\section{Discussão}

A educação superior brasileira tem enfrentado alguns problemas nas últimas décadas, como qualificar os educandos para exercer adequadamente sua profissão (Santos, 2010). Buscando melhorar a formação, utiliza-se da extensão universitária como instrumento para efetivação do seu compromisso social, trazendo benefícios para os aprendizes e para a comunidade (Rodrigues et al., 2013). A UEM fomenta a extensão, intensificando as relações transformadoras entre a Universidade e Sociedade por meio dos seus processos educativo, cultural e científico (UEM, 2017). No entanto, para aprimorar a extensão universitária são necessários processos avaliativos rigorosos e sistemáticos para que as ações extensionistas, como o Curso Qualificação em Gestão de Saúde Bucal no SUS, exerçam seu papel de transformar a situação social e formação de indivíduocidadão com pensamento crítico-reflexivo voltado para sua realidade (Arroyo \& Rocha, 2010).

É importante ressaltar a diferença entre invenção e inovação: a primeira ocorre no momento que a ideia é gerada; e a segunda ocorre quando a ideia é implementada no meio social (Schumpeter, 1982). Neste estudo, a inovação foi compreendida como a produção e circulação do conhecimento (Andrade, 2006), educação e capacitação da força de trabalho (Cardoso, 1997), além da busca, experimentação e adoção de novos processos (Serzedello \& Tomaél, 2010). Considerou-se que a inovação pode ser uma alteração de sentido a respeito da prática corrente e deve ter o caráter intencional, sistemático e planejado, em oposição às mudanças espontâneas, propiciando benefícios para a comunidade e auxiliando no desenvolvimento socioeconômico do país (Messina, 2001).

A partir dos conceitos de inovação adotados neste estudo (Brasil, 2018; Rogers, 2003; Schumpeter, 1982; Messina, 2001), o curso foi considerado um processo inovador, tendo sido reconhecido pelo Ministério da Saúde, com o apoio da Organização Pan-Americana da Saúde/Organização Mundial da Saúde, por meio da premiação do Laboratório de Inovação em 
Educação na Saúde com foco em Educação Permanente em Saúde, que selecionou 15 experiência em todo o país, dentre diversas iniciativas na área da saúde. Verificou-se que o Curso pôde contribuir para a melhoria do processo de trabalho, qualificação de profissionais e melhoria da gestão, tendo o objetivo de atender as necessidades da população e da qualificação da atenção à saúde no SUS (Brasil, 2018). Além disso, este trabalho foi reconhecido pela Sociedade Brasileira de Pesquisa Odontológica - a divisão brasileira da International Association for Dental Research (IADR)-, tendo recebido o primeiro lugar no Prêmio de Pesquisa Odontológica de Ação Coletiva no ano de 2018, pela utilização da metodologia problematizadora na educação profissional em serviço. Considerando que o curso implementado no Paraná agregou valor aos profissionais e serviço, beneficiando a comunidade, a divulgação desta proposta e sua amplitude são pontos importantes para serem estudados e detalhados.

O espalhamento geográfico desta inovação, pôde ser verificado pela adesão gradativa e crescente dos profissionais de diferentes localidades do Estado do Paraná. No presente estudo, 321 municípios (80\%) aderiram a pelo menos um ciclo do Curso, apresentando semelhança com o estudo de Pankratz et al. (2002), que avaliou 214 distritos escolares em 12 estados dos Estados Unidos em relação à adesão de uma política federal de prevenção às drogas. No estudo americano, $82 \%$ relataram ter concluído pelo menos um ciclo e $68 \%$ os dois ciclos. No presente estudo a adesão a dois ciclos foi de $40 \%$. Uma possível diferença pode ter sido pelas necessidades locais e priorização da gestão, o que resulta numa adesão expressiva.

A adesão de 22 ( $\left.1^{\circ} \mathrm{ciclo}\right), 384$ ( $\left.2^{\circ} \mathrm{ciclo}\right), 262\left(3^{\circ} \mathrm{ciclo}\right)$ e 636 ( $\left.4^{\circ} \mathrm{ciclo}\right)$ profissionais nos quatros ciclos respectivamente, estão de acordo com a teoria de taxa de difusão de inovação de Rogers (2003), que mostra que a difusão de políticas é cumulativa no tempo e estabelece um modelo que assemelha-se a curva com formato S. No início a inovação atravessa um período de lentidão, tendo uma baixa adesão de adotantes denominados inovadores ou precursores. Nesta etapa as regionais de saúde e municípios melhor estruturados tendem a aderir ao processo com maior facilidade. Posteriormente, um gradual crescimento, antes de experimentar um período de crescimento relativamente acentuado. A teoria também estabelece que, seguindo o período de rápido crescimento a taxa de adoção da inovação, irá gradualmente se estabilizar e eventualmente declinar-se, o que não foi possível verificar com este curso, pois após o quarto ciclo, não houve mais a oferta deste até o momento. Esta elevada adesão mostra a característica da compatibilidade, definida por Rogers (2003) como sendo o grau em que uma inovação é percebida como consistente e necessária aos adotantes potenciais. Assim, fica claro que os profissionais de saúde enxergaram a necessidade de qualificação do serviço a partir do conhecimento de experiências prévias de outros municípios nas etapas iniciais do processo de capacitação, melhorando o atendimento ofertado à população de seu município, seguindo os valores e missão do SUS.

Os elementos condicionantes da difusão de inovação apresentados na Tabela 1 demonstraram que estes foram fatores facilitadores da taxa de distribuição deste processo. O primeiro elemento condicionante destacado é a vantagem relativa, ou seja, o grau em que a inovação é percebida como sendo melhor do que as práticas atuais e está positivamente relacionada ao seu índice de adoção. Pela elevada adesão voluntária ao Curso (80\%), os profissionais perceberam a necessidade de qualificação, e que esta ocorreu gradualmente durante o processo de educação permanente, tendo momentos de trocas entre os participantes. Além de ter oferecido ferramentas para realização do diagnóstico e planejamento local, podendo desta forma, transformar as práticas de trabalho e desenvolver o pensamento crítico reflexivo. Para D’Ávila et al. (2014), a adesão à realização da Educação Permanente em Saúde pelos profissionais é um desafio, sendo esta uma estratégia que possibilita transformações das práticas no processo de trabalho, devendo estar centrado nas necessidades de saúde e especificidades locais.

A observabilidade é caracterizada como sendo o grau com que os resultados de uma inovação são visíveis por outros (Rogers, 2003). Neste estudo considerando que os alunos de um ciclo poderiam se tornar multiplicadores nos ciclos seguintes, este Curso traz a potencialidade loco-regional, sendo que os profissionais que realizaram o Curso possam ser influência para os 
demais dos municípios próximos, causando um espalhamento por contiguidade. Raiher e Lima (2010) mostraram que no Estado do Paraná a difusão do desenvolvimento social e econômico ocorre por contiguidade, seguindo redes de cidades interligadas por estradas e pelo próprio sistema de saúde, organizado em regionais e suas sedes. As localidades desenvolvidas localizam-se nos extremos do Estado, e que as subdesenvolvidas localizam-se na região Central, o que difere do presente estudo, no qual o extremo Oeste teve a menor distribuição geográfica e a Região Central teve a maior. Estes dados demonstram que o Curso conseguiu difundir-se por áreas que mais necessitam de políticas públicas para melhorar as condições de vida desta população, gerando satisfação de profissionais, equipe e usuários. Além disso, verificou-se que todos os municípios com baixo IDH-M tiveram acesso e aderiram ao Curso, fato este que promoveu a equidade, transpassando as barreiras do desenvolvimento social e econômico local.

Outro resultado relevante é a diversidade e quantidade de atores que participaram da construção e realizaram a capacitação no Estado do Paraná (Coordenador Geral, Estadual, Regionais e Municipais de Saúde Bucal, CD, ASB, TSB, ACS, dentre outros profissionais da saúde).

A condição de saúde bucal da população não é resultado apenas das práticas odontológicas, mas sim das construções sociais realizadas pelos seres humanos, incluindo os profissionais de saúde (Narvai \& Frazão, 2006). Verificou-se neste Curso, que houve uma participação progressiva nos ciclos, iniciando com os gestores da saúde bucal, incluindo a equipe de saúde bucal e ampliando a discussão para outros profissionais da saúde, o que tornou este Curso relevante pelas construções coletivas, integradas e ampliadas de intervenções em benefício de uma população adscrita. Mockedeci et al.(2014) demonstram que em um estudo com ACS em Juiz de Fora, Minas Gerais, 33\% relatam ter dificuldade em realizar suas atribuições, incluindo temas de saúde bucal, e 15\% mencionaram que as atividades de saúde bucal não são atribuições dos ACS e que estes desconhecem a prevalência das doenças bucais e suas implicações sobre a vida do indivíduo. Dentro desta perspectiva, a participação de diversos profissionais realizando o diagnóstico de gestão local contido no TCC, planejando ações para qualificar o sistema e implementando intervenções, fomentou pontos de vistas diversos e uma visão holística dos processos de trabalho, do cuidado e estrutural e a valorização da saúde bucal. Além disso, oportunizou o trabalho interprofissional, a discussão de indicadores de saúde e o planejamento futuro, ampliado, permitindo que a gestão fosse ascendente, por meio de registros sistemáticos.

Pensando na dinamicidade do processo saúde-doença e que este está inserido na transformação social, econômica, política e cultural de determinado território, a oferta do Curso em ciclos de capacitação ofereceu a oportunidade de o mesmo profissional realizá-lo em mais de um ciclo. Além disso, foi possível atualizar o diagnóstico, realizar um novo planejamento, visto que a realidade já não seria a mesma do primeiro momento. Assim, o processo educativo deve ser permanente, uma vez que o SUS é dinâmico e a realização uma única vez do Curso não significa a apropriação de todos os temas abordados, além de permitir um monitoramento das propostas implementadas. Para poder dar conta dos problemas complexos da sociedade, há a necessidade de qualificação permanente, pois segundo a curva de aprendizagem, à medida que as repetições são efetuadas, os profissionais demandam menos tempo para a execução das atividades e exercem com melhor qualidade, seja pela familiaridade, seja pela adaptação às ferramentas utilizadas (Dar-El, 2000). Considerando o SUS um sistema de saúde novo, em fase de consolidação e construção de políticas públicas mais efetivas, pode-se compreender a necessidade de apropriação de seus princípios filosóficos, bem como de estabelecer práticas diárias eficazes para a promoção da saúde da população.

Um aspecto a ser discutido também é o papel das Regionais de Saúde, sendo representada por seus Coordenadores Regionais de Saúde Bucal, que podem ser considerados polos de difusão para os municípios, visto que treze Regionais tiveram mais de $90 \%$ de seus municípios em pelo menos um ciclo. Em contrapartida, as Regionais de Cascavel e Toledo tiveram menos de 30\% dos municípios que aderiram a pelo menos um ciclo. Este ponto é evidenciado no estudo Valaitis et al. (2016) que afirma que a liderança organizacional é fator de influência na adoção de políticas públicas, podendo ser uma barreira ou 
um facilitador do processo, mostrando que os esforços dos agentes de mudança têm papel relevante na difusão hierárquica. Assim, o preparo de gestores e lideranças também é um desafio para o sistema de saúde, para a difusão de processos inovadores como este, já que todas as Regionais tiveram o mesmo acesso à formação de multiplicadores. Sugere-se investimentos e a priorização na formação de gestores e lideranças para uma melhor condução e implementação de novas políticas.

A experimentabilidade, definida por Rogers (2003) como sendo o grau com que uma inovação pode ser usada experimentalmente antes de ser adotada ou rejeitada, pode ser verificada através da metodologia do Curso, que o aluno de um ciclo pode tomar a decisão de multiplicá-lo no ciclo seguinte. O Curso de Qualificação da Gestão do SUS em Saúde Bucal teve nos quatro ciclos 95 multiplicadores voluntários que capacitaram 1304 profissionais. Fernandes et al. (2007) em seu estudo sobre educação na epilepsia, relata que inicialmente 11 médicos foram capacitados, e posteriormente multiplicaram para 810 profissionais da saúde, incluindo trabalhadores da atenção primária, demonstrando que a utilização de multiplicadores permite uma expansão rápida e eficaz das informações. Desta maneira, é possível aproveitar as potencialidades existentes no quadro de servidores para que estes experimentem e possam aderir à proposta, compreendendo que cada um tem algo a aprender e algo a ensinar, sendo a troca de conhecimentos, experiências muito enriquecedoras.

Vale ressaltar sobre os canais e as formas de comunicação entre a Coordenação do Curso/Regionais e entre Regionais/Municípios, que foram: comunicação interpessoal, divulgação em sites institucionais da SESA e da UEM e comunicação virtual por meio de e-mails institucionais, o que garantiu a divulgação do curso e a adesão de municípios dispersos e menos conectados por estradas. Para cada início de ciclo, foram realizados encontros presenciais da coordenação com os representantes da SESA e Coordenadores Regionais, para pactuar metas, ouvir as demandas locais e realizar as alterações necessárias na condução do Curso, já que a relação interpessoal tem mostrado aspectos positivos mais efetivos do que um vídeo ou um ofício (Parcel et al., 1995). Desta forma, se faz necessário Coordenadores que exerçam o protagonismo em sua Regional e que também desenvolvam habilidades de comunicação e articulação, para incentivar os profissionais e ouvilos, sensibilizando-os para a importância da qualificação da gestão.

Em relação à complexibilidade da inovação, esta refere-se a quanto uma inovação é percebida como relativamente difícil de ser entendida e utilizada (Rogers, 2003). Desta forma, o instrumento diagnóstico utilizado como norteador para o estudo e compreensão da dimensão da gestão é uma ferramenta complexa, mas acessível a todos os trabalhadores. Esta foi baseada em três pilares importantes para a gestão da saúde bucal: a gestão estrutural, a gestão do cuidado e do trabalho, que sustentam a qualidade da atenção à saúde bucal. O instrumento de diagnóstico foi construído com a finalidade de ajudar o gestor, a equipe de saúde e a comunidade a trabalharem juntos para uma atenção à saúde bucal resolutiva e de qualidade, com o entendimento de que a gestão depende do empenho de cada um (trabalhador e usuário) e que todos são atores sociais no processo de melhoria do nosso país. Este Curso também apresentou baixa complexidade na questão de investimentos financeiros, uma vez que os custos envolvidos na implementação desse Curso foram baixos, seja por usar material didático digital, multiplicadores voluntários ou pelo fator de que muitas intervenções propostas pelos alunos não necessitarem de recursos por lidar com tecnologias leves. No entanto, existiram barreiras para implementação, como o apoio dos gestores dos municípios e as características individuais dos profissionais.

Por fim, pode-se perceber que o sistema social favoreceu a difusão da inovação através da parceria e apreciação das etapas de desenvolvimento do Curso, desde a construção do instrumento de diagnóstico até a elaboração do TCC, pela Coordenação Nacional de Saúde Bucal do Ministério da Saúde e da Secretaria Estadual de Saúde Bucal. O alinhamento conceitual entre gestores das esferas federal, estadual, regional, municipal e local, parece ser um dos pontos fortes desta proposta, já que a compreensão de um norte comum entre gestores, profissionais e comunidade é o caminho para construção de uma sociedade melhor. 


\section{Conclusão}

Com a realização dessa pesquisa, conclui-se que o Curso de Qualificação da Gestão do SUS em saúde bucal foi um processo inovador em políticas públicas, tendo em vista os seguintes aspectos: fortalecimento da relação ensino-serviço; criação de um instrumento norteador para a avaliação da gestão em Saúde Bucal com alinhamento local, estadual e federal; a reprodutibilidade em outras localidades; utilização de tecnologia de informação e comunicação e de acesso livre na internet; valorização da interprofissionalidade; criação de espaços de interação entre municípios, equipes e gestores e empoderamento de lideranças onde o aprendente em um ciclo pode se tornar o multiplicador no seguinte.

Verificou-se que houve uma alta cobertura da distribuição espacial do processo educativo, com a participação de 321 municípios ( $80 \%$ dos municípios do Paraná) ao final de 4 ciclos, em 8 anos de processo educativo. A adesão foi heterogênea no tempo e no espaço, mostrando que os profissionais aceitam em tempos diferentes as mudanças. O padrão de distribuição espacial dos municípios e profissionais ocorreu em um processo hierárquico e por contiguidade, demonstrando o papel importante das Regionais de Saúde do Paraná.

Os coordenadores regionais e municipais de saúde bucal operaram como elementos multiplicadores da inovação, potencializando o número de profissionais qualificados para uma gestão mais eficaz da saúde bucal no SUS. Além disso, as parcerias com as 22 Regionais de Saúde (100\%) e com as Coordenações Estadual e Federal de Saúde Bucal mostraram que a construção coletiva e o norte comum foram pontos chaves em todo o processo, mostrando a importância da integração ensinoserviço.

Apesar dos resultados promissores apresentados da qualificação da Gestão do SUS em saúde bucal, sugere-se novos estudos para avaliar os impactos diretos nos serviços de saúde bucal e na qualidade de vida da população, por meio de indicadores odontológicos, bem como quantificar e analisar dados que possam comprovar se houve difusão de inovação neste processo educativo. Vale ressaltar que existe um grande potencial de ampliação desta proposta, já que o número alcançado de profissionais de saúde em todo o Estado do Paraná foi expressivo por ser um processo em construção, mas limitado, considerando o número total de trabalhadores do SUS. Além disso, cada participante é convidado a participar deste Curso em novos momentos para o aprofundamento do estudo, atualização das normas vigentes, desenvolvimento de metas e estratégias em equipe, realizando a transposição didática dos conteúdos estudados e das normas e diretrizes do SUS, que é um sistema de saúde novo, dinâmico, que necessita de profissionais abertos, sensíveis, éticos, responsáveis, comprometidos e motivados à mudança, para dar respostas às necessidades da população e promover saúde.

Por fim, o Curso de Qualificação da Gestão do SUS em Saúde Bucal, coordenado pela Universidade Estadual de Maringá, auxiliou na compreensão e organização das redes de atenção à saúde, na melhoria do processo de trabalho no SUS, na motivação e entendimento do papel do trabalhador, estimulando a educação permanente e a promoção de saúde, na tentativa de buscar a garantia do cidadão brasileiro ao direito à saúde bucal.

\section{Agradecimentos}

Os autores gostariam de agradecer ao Conselho Nacional de Desenvolvimento Científico e Tecnológico - CNPq (Proc. No 401514 / 2013-7), Ministério da Saúde do Brasil - MS (Convênio nº 1.333 / 2010), Secretaria de Saúde do Estado do Paraná pelo suporte fornecido no desenvolvimento e aplicação deste instrumento. Este estudo foi financiado em parte pela Coordenação de Aperfeiçoamento de Pessoal de Nível Superior - Brasil (CAPES) - Código Financeiro 001. Agradecemos aos Professores Gilberto Alfredo Pucca Júnior, Raquel Sano Suga Terada e Luiz Fernando Lolli; à Dra. Josely Emiko Umeda e Dra. Tânia Harumi Uchida pela inestimável contribuição na criação e condução do processo de Qualificação da Gestão do SUS em Saúde Bucal no Estado do Paraná. Aos Coordenadores Estaduais de Saúde Bucal Dr. Léo Kriger, Dr. Guilherme Grazziani e Dra. Erika Feller, aos gestores e equipes de saúde dos municípios do Paraná pela parceria e colaboração. 


\section{Referências}

Andrade, T. N (2006). Aspectos sociais e tecnológicos das atividades de inovação. Lua Nova: Rev Cult e Polit, 66, $139-166$.

Arroyo, D. M. P., \& Rocha, M. S. P. M. L (2010). Meta-avaliação de uma extensão universitária: Estudo de caso. Revista da Avaliação da Educação Superior, 15(2),131-157.

Bessant, J., Lamming, R., Noke,H., \& Phillips, W (2005). Managing innovation beyond the steady state. Technovation. 25(12), 1366-1376.

Brasil (2004). Lei 10.861, de 14 de abril de 2004. Institui o Sistema Nacional de Avaliação da Educação Superior - SINAES e dá outras providências. Diário Oficial da União.

Brasil (2006). Ministério da Saúde. Secretaria de Atenção à Saúde. Departamento de Atenção Básica. Cadernos de Atenção Básica: saúde bucal, Brasília, DF.

Brasil (2018). Ministério da Saúde. Laboratório de Inovação em Educação na Saúde com ênfase em Educação Permanente / Ministério da Saúde, Organização PanAmericana da Saúde / Organização Mundial Saúde no Brasil. - Brasília: Ministério da Saúde.

Cardoso, A. P (1997). Educação e inovação. Revista Millenium, 6, 1-9.

Christensen, C., Curtis, W. J., \& Horn, M. B (2012). Disrupting class:How disruptive innovation will change the way the world learns. McGraw Hill.

Dar-El, E (2000). Human Learning: from Learning Curves to Learning Organizations. Springer.

D'avila, L. S., Assis, L. N., Melo, M. B., \& Brant, L. C(2014). Adesão ao Programa de Educação Permanente para médicos de família de um Estado da Região Sudeste do Brasil. Ciênc. saúde coletiva. 19(2), 401-416, 2014.

Duarte, L. S., Pessoto, U. C., Guimarães, R. B., Heimann, L. S., Carvalheiro, J. R., Cortizo, C. T., \& Ribeiro, E. A. W (2015). Regionalização da saúde no Brasil: uma perspectiva de análise. Saude soc., 24 (2), 472-485.

Fernandes, P. T., Noronhal, A. L. A., Sander, J. W., Bell, G. S., \& Li, M. L (2007). Training the trainers and disseminating information: a strategy to educate health professionals on epilepsy. Arq. Neuro-Psiquiatr., 65 (supl. 1), 14-22.

Frazão, P., \& Narvai, P. C (2009). Saúde bucal no Sistema Único de Saúde: 20 anos de lutas por urna política pública. Saúde em Debate, 33 (81), 64-71.

Fujimaki, M., Terada, R. S. S., Lolli, L. F., Galbiati, C. F., Umeda, J. E., Uchida, T. H., \& Antoniassi, C. P (2019). Qualificação da Gestão do SUS em saúde bucal no Paraná. In: DITTERICH, Rafael Gomes et al. Caminhos e trajetórias da saúde bucal no estado do Paraná. Londrina: INESCO, $179-196$.

Galbiati, C. F (2018). Qualificação da gestão e da equipe de saúde bucal por meio da implementação da educação permanente em saúde no estado do Paraná. Tese de Doutorado em Odontologia Integrada - Universidade Estadual de Maringá, Maringá (PR).

Giacomini Filho, G., Goulart, E. E., \& Caprino, M. P (2007). Difusão de inovações: apreciação dos estudos de Rogers. Revista FAMECOS, 14 (33), $41-45$.

Instituto Brasileiro de Geografia e Estatística (IBGE) (2012). Censo Brasileiro de 2010.

Instituto Paranaense de desenvolvimento econômico e social (IPARDES) (2010). Indicadores de desenvolvimento humano municipal.

Kusma, S. Z., Moyses, S. T., \& Moyses, S. J (2012). Promoção da saúde: perspectivas avaliativas para a saúde bucal na atenção primária em saúde. Cad. Saúde Pública, 2, supl. p. s9-s19.

Masseto, M (2004). Inovação na Educação Superior. Interface (Botucatu), 8 (14), 197-202.

Messina, G (2001). Mudança e inovação educacional: notas para reflexão. Cad. Pesqui, 114, 225-233.

Mockdeci, H. R., Souza, T. S., Rodrigues, C. M., \& Leite, I. C. G (2014). Capacitação em saúde bucal de Agentes Comunitários em Juiz de Fora- MG: a efetividade do programa. HU Revista, 9 (3 e 4), 45-52.

Moore, G. C., Benbasat, I (1991). Development of an instrument to measure the perceptions of adopting an information technology innovation. Information Systems Research, 2 (3), 192-222.

Morgenstern, H. (1995) Ecologic studies in Epidemiology: Concepts, principles, and methods. Annu. Rev. Public Health 16, 61-81.

Narvai, P. C., \& Frazão, P (2006). Epidemiologia, política, e saúde bucal coletiva. In: Antunes J.L.F, Peres M.A. Epidemiologia da saúde bucal. Rio de Janeiro: Guanabara Koogan, p.441.

Pankratz, M., Hallfors, D., \& Cho, H (2002). Measuring perceptions of innovation adoption: the diffusion of a federal drug prevention policy. Health education research, 17 (3), 315-326.

Parcel, G., O'Hara-Tompkins, N. M., Harrist, R. B., Basen-Engquist, K. M., McCormick, L. K., Gottlieb, N. H., Eriksen, M. P (1995). Diffusion of effective tobacco prevention program. Part 2: Evaluation of the adoption phase. Health Education Research, 10, $297-307$.

PROEX (2012). Políticas Nacional de Extensão Universitária Brasileira. Editora UFMG.

Raiher, A. P., \& Lima, J. F (2010). A dispersão espacial do desenvolvimento econômico: o caso dos municípios paranaenses. Revista de desenvolvimento econômico. 12 (21), 84-99. 
Research, Society and Development, v. 10, n. 8, e34410817460, 2021

(CC BY 4.0) | ISSN 2525-3409 | DOI: http://dx.doi.org/10.33448/rsd-v10i8.17460

Rangel-S, M. L., Barbosa, A. O., Riccio, N. C. R., \& Souza, J. S (2012). Redes de aprendizagem colaborativa: contribuição da Educação a Distância no processo de qualificação de gestores do Sistema Único de Saúde - SUS. Interface (Botucatu), 16 (41), 545-556.

Rodrigues, A. L. L., Prata, M. S., Batalha, T. B. S., Costa, C. L. N. A., P\& assos Neto, I. F (2013). Contribuições da Extensão Universitária na Sociedade. Cadernos de Graduação - Ciências Humanas e Sociais. 1 (16), 141-148.

Rogers, E. M (2003). Diffusion of innovations. (5th ed). Free Press.

Santos, W. J. L (2010). Financiamento e investimento da educação nos municípios de Duque de Caxias e de Nova Iguaçu - RJ: avaliação da eficácia, da efetividade e da transparência das políticas públicas. 300 f. Tese (Doutorado) - Universidade Estadual do Rio de Janeiro, Faculdade de Educação, Rio de Janeiro, RJ, Brasil.

Secretaria Estadual de Saúde do Paraná (SESA) (2018). Regionais de Saúde.

Serzedello, N. T. B., \& Tomael, M. I (2010). Produção tecnológica: um diagnóstico da área de ciências agrárias da Universidade Estadual de Londrina (UEL). In: Curty RG. Produção intelectual no ambiente acadêmico. UEL/CIN, 69-88.

Schumpeter, J. A (1982). Teoria do desenvolvimento econômico. Abril Cultural, (Os economistas).

Tsuzuki, F. M. (2017). Avaliação da percepção de multiplicadores sobre o processo de qualificação da gestão do SUS em saúde bucal no Paraná. Relatório de pesquisa do Programa de Iniciação Científica da Universidade Estadual de Maringá (Odontologia) - UEM.

Universidade Estadual de Maringá (UEM) (2017). Regulamento para o desenvolvimento de projetos de extensão na Universidade Estadual de Maringá, Resolução 033/2017.

Valaitis, R., MacDonald, M., Kothari, A., O’Mara, L., Regan, S., Garcia, J., Murray, N., Manson, H., Peroff-Johnston, N., Bursey, G., \& Boyko, J (2016). Moving towards a new vision: implementation of a public health policy intervention. BMC Public Health, 16, 412. 